Results: As a cohort, there was no significant difference in postexercise fetal fraction $(p=0.38)$. However, women with BMI $<25$ had an average fetal fraction change of $-2.3 \%(n=5, p=0.02)$, while women with BMI $\geq 25$ had an average change of $+0.5 \%$ $(n=10, p=0.25)$. Change in fetal fraction was not influenced by gestational age, maternal age, change in heart rate, or change in systolic blood pressure.

Discussion: Moderate maternal exercise may only impact fetal fraction when maternal BMI is $<25$. Further studies are required to confirm this association.

\section{MEASURABLE RESIDUAL DISEASE (MRD) DETECTION BY NEXT GENERATION SEQUENCING (NGS) IN ACUTE LYMPHOBLASTIC LEUKAEMIA (ALL)}

Wendi Lin $^{1}$, Rishu Agarwal ${ }^{2}$, Suzanne Svobodova ${ }^{2}$, Chun Yew Fong

${ }^{1}$ Clinical Haematology, Austin Health, Melbourne, Australia; and ${ }^{2}$ Molecular Diagnostics, Austin Pathology, Melbourne,

Australia

Introduction: MRD, a key prognostic factor in ALL, is traditionally assessed by flow cytometry and/or allele specific oligonucleotide PCR (ASO-PCR). Here we describe validation and implementation of novel NGS technologies to measure MRD at clinically relevant time points.

Methods: Extracted DNA from bone marrow were analysed using the LymphoTrack ${ }^{\circledR}$ Diagnostic Assay Panel to detect $\operatorname{IgH}$ gene rearrangements. Sequence clonality was defined as $>2.5 \%$ of total reads and $>2 \mathrm{x}$ frequency of the third most frequent sequence. A 100 cell equivalent spike-in control (LymphoQuant ${ }^{\mathrm{TM}}$ ) was utilised for normalisation.

Results: Results were concordant $(78.18 \%)$ between assay methods with the exception of 12 samples. In 10 cases, MRD was detected by NGS at a lower level than flow cytometry and ASOPCR, which were negative. MRD positivity by NGS corresponded with poor clinical outcomes in these patients. We demonstrate that the use of spike-in controls deliver clinically meaningful results. Conclusion: MRD detection by NGS is complementary to testing using flow cytometry and ASO-PCR. NGS has the potential advantage of increased sensitivity, detection of clonal evolution and a rapid turnaround time. Normalisation of MRD levels to cell equivalents is required to suitably compare results with flow cytometry and ASO-PCR.

\section{REVIEW OF 12 MONTHS OF COPY NUMBER VARIANT CALLING ON A CLINICAL NEXT GENERATION SEQUENCING PIPELINE}

Dylan A. Mordaunt ${ }^{1,2}$, Julien Soubrier ${ }^{1}$, Song Gao ${ }^{1}$, Lesley Rawlings $^{1}$, Jillian Nicholl ${ }^{1}$, Sui $\mathrm{Yu}^{1}$, Janice Fletcher ${ }^{1}$, Karin Kassahn

${ }^{1}$ Genetics and Molecular Pathology, SA Pathology, SA, Australia; and ${ }^{2}$ Department of Paediatrics, School of Medicine, University of Adelaide, SA, Australia

Background: Next generation sequencing (NGS) provides the opportunity to detect not only single nucleotide variants and small indels, but also larger whole exon or whole gene deletions and duplications (CNVs).
Method: We implemented CNV calling in our NGS pipeline utilising the DECoN, XHMM and PanelCNV packages. We compared the performance with multiplex ligation-dependent probe amplification (MLPA) on our 1200 gene panel (R1kD) and with chromosomal microarray (CMA) on our whole-exome (WES) platform.

Results: On the R1kD, we reviewed 197 patient cases which had both NGS and MLPA. We detected 25 deletions and 7 duplications, all of which were detected by both methods. Seven other CNV calls from NGS were considered artefacts during IGV review due to the presence of pseudogenes and poor mapping. On the WES platform, we reviewed 37 patient cases which had both NGS and CMA and detected 11 deletions and 10 duplications by both methods. NGS called some additional smaller events which were not reported by CMA.

Conclusions: CNV analysis on NGS can be implemented in clinical pipelines. Based on review of the first 12 months of service we have moved some tests to NGS with MLPA only performed to check positive results.

\section{HEPARIN INDUCED THROMBOCYTOPENIA IN PATIENTS WITH SEVERE RHABDOMYOLYSIS: A CASE SERIES}

Roya Arabi ${ }^{1,2}$, Jessica Margaret Heenan ${ }^{1}$, Julia Helen Gardner ${ }^{1}$, Muhajir Mohamed ${ }^{1,3}$

${ }^{1}$ Launceston General Hospital, Launceston, Tas, Australia;

${ }^{2}$ Royal Hobart Hospital, Hobart, Tas, Australia; and

${ }^{3}$ University of Tasmania, Launceston, Tas, Australia

Heparin-induced thrombocytopenia (HIT) is a known immunological complication of heparin administration which can cause life threatening thrombosis. Here, we report a series of three cases of HIT in the context of acute kidney injury due to rhabdomyolysis that presented to Launceston General Hospital, Tasmania, between December 2017 and December 2018. During this time, there were a total number of four patients with HIT, out of which three had rhabdomyolysis. Patients were all men with age between 29 and 68 years old. The causes of rhabdomyolysis were statin therapy (in two cases) and ischemia induced by compartment syndrome (in one case). All patients received unfractionated heparin and continuous renal replacement therapy for 2-11 days. 4Tscore was 4 in one patient and 5 in two other patients. None of patients had thrombosis complication. In all patients, anti-heparin/PF4 antibody was detected by BioRad Heparin/PF4 gel column assay and the diagnosis was confirmed by serotonin release assay. Patients received non-heparin anticoagulation. Platelet count recovered after cessation of heparin. Since rhabdomyolysis is an uncommon condition, the concomitant development of HIT in our 3 cases raises the suspicion whether administration of unfractionated heparin in patients with rhabdomyolysis poses an additional risk for developing HIT.

\section{IDARUCIZUMAB RESISTANCE DUE TO A MARKEDLY ELEVATED DABIGATRAN CONCENTRATION - A CASE REPORT}

\footnotetext{
H. Cashman ${ }^{1,2}$, J. Joseph ${ }^{1,2}$, D. Roberts ${ }^{1,2,3}$

${ }^{1}$ Department of Haematology, St Vincent's Hospital, Sydney, Australia; ${ }^{2}$ University of New South Wales, Sydney, Australia;
} 